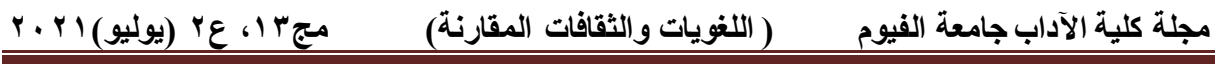

\title{
Traduction des chansons dans le film Pocahontas de Disney entre adaptation et musicalité
}

Samah Hassan Abdou Nasr ${ }^{*}$

samah.hassan@women.asu.edu.eg

\section{Résumé}

Dans cet article, nous cherchons à découvrir la traduction des chansons du film de dessin animé de Disney Pocahontas en français et en arabe égyptien. Pour quelle raison choisir le dialecte égyptien pour la traduction arabe? Quels sont les enjeux de la traduction des films de Disney pour leur commercialisation en Orient ? Entre dialecte et arabe classique, quel niveau pour quel public ? Il est également question de découvrir les procédés de transfert des références culturelles : les connotations (nommer la différence de races par exemple), la valeur morale de quelques phénomènes culturels spécifiques (patrimoine culturel), le sacré, etc.

Mots-clefs : Traduction audiovisuelle- dessins animésadaptation- identité - doublage.

* Professeur-adjoint à l'Université Ain Shams, Faculté de Jeunes Filles

(Traduction des chansons dans le film...) Dr. Samah Abdou Nasr. 


\section{Introduction}

La traduction des chansons des films de Disney en français et en arabe égyptien est assez intéressante au niveau culturel. Quels sont les impératifs qui imposent au traducteur certains choix en vue d'assurer la bonne réception du film? Face à cette situation, les traducteurs suivent quelles stratégies? Tirées d'une même version anglaise originale et doublées en deux langues différentes, les chansons ont en effet recours à des structures variées qui cherchent à suivre le rythme original en se pliant aux rimes appréciables dans les langues cibles.

Notre corpus est extrait du film Pocahontas, produit en 1995 par Walt Disney Pictures, que nous avons choisi grâce à son histoire inspirée de la vie d'une femme amérindienne. Dans ce film de dessins animés, les chansons doublées permettent de développer des réflexions sur plusieurs points qui reflètent une attention particulière aux pensées de deux personnages qui ont deux visions différentes du monde. Ce duel superpose deux prises de positions entre deux groupes: les Amérindiens et les explorateurs britanniques ou colons. Les chansons jouent un rôle essentiel dans la présentation de ces deux groupes dès la séquence d'ouverture et tout au long du film. Nous avons transcrit les versions arabes pour pouvoir les comparer aux chansons originales en anglais dont les transcriptions étaient disponibles sur le site de Disney et la version française sur internet également.

(Traduction des chansons dans le film...) Dr. Samah Abdou Nasr. 


\section{Enjeux culturels du doublage des chansons}

Les chansons sont plus faciles à retenir et influencent fortement les spectateurs. Le support audiovisuel use de divers moyens pour maximiser les effets sur l'audience et toucher leurs émotions : l'image, la musique et les paroles. En fait, les thèmes des chansons sont motivés par la prise de position de la société Disney qui sert à présenter le patrimoine des anciens habitants de l'Amérique ou les propriétaires autochtones de cette terre appelée le Nouveau monde aux yeux des colons. Le film met en premier lieu la mise en avant des valeurs humaines dont le respect de chaque être humain, le respect de la nature et le maintien de la paix.

Cette étude cherche à découvrir les procédés de transfert des références culturelles : les connotations (nommer la différence de races par exemple), la valeur morale de quelques phénomènes culturels spécifiques (patrimoine culturel), le sacré, etc. La médiatisation de la culture indienne à partir du personnage féminin de POCAHANTAS et le contexte de la conquête du Nouveau monde pour chercher l'or. L'héritage culturel des Amérindiens et la vision des explorateurs sont opposés tout au long du film. Notre analyse porte sur les étapes de rencontre des deux cultures à travers les chansons du film. L'examen de la traduction des chansons montre des choix divers pour lesquels le traducteur opte dans son travail. Il essaye d'adapter les mots au

(Traduction des chansons dans le film...) Dr. Samah Abdou Nasr. 
rythme musical et aux mouvements des lèvres des personnages ; dans cette démarche plusieurs contraintes sont mises en jeu. Au niveau de la traduction, l'adaptateur d'un film oscille entre deux tendances : «préserver la couleur locale, pour respecter la cohérence image-son (..) ou au contraire acclimater ces références pour les rendre accessibles au public cible, en franchissant délibérément la double frontière de la langueculture.» Brisset (2017: 36) Alors qu'au niveau de la synchronisation des paroles à l'image, ses choix sont guidés par les principes phonétiques qui président à la musicalité des fins de phrases en fonction de la longueur et des groupes rythmiques.

Pour illustrer nos propos, les exemples sont extraits de trois chansons principales dans le film qui marquent trois temps forts du récit : la chanson au début du film pour présenter le groupe de colons qui travaillent dans la Virginia Company, la chanson qui présente le groupe des autochtones intitulée «Au son calme du Tam Tam » et la chanson qui met en scène le point culminant du conflit entre les deux groupes, celle « Des sauvages». Ces chansons représentent une critique de l'ethnocentrisme occidental représenté par les colons britanniques. A travers l'emploi du mot sauvage, chaque personnage refuse d'accepter l'autre. Nous aborderons les différentes désignations de l'autre et les procédés d'adaptation dans les deux versions française et anglaise.

(Traduction des chansons dans le film...) Dr. Samah Abdou Nasr. 


\section{L'image de l'autre}

Dans cette partie, il s'agit d'explorer le lexique utilisé pour se présenter et présenter l'autre dans les chansons. Au niveau chronologique, les chansons présentent d'abord chaque groupe seul dans des séquences séparées, puis la rencontre personnelle entre les deux protagonistes représentant deux visions du monde et enfin la chanson du conflit opposant les deux groupes différents sur le plan ethnique. Le rôle de l'adaptation étant de créer un texte qui sera chanté, quels sont les mots à choisir et quelle sonorité préférer en conformité avec le destinataire du texte et suivre un rythme approprié.

\subsection{La présentation de deux groupes opposés}

Le titre du dessin animé introduit dans la vie des Amérindiens en pleine nature en toute sécurité. Puis, des marins britanniques faisant partie de la Virginia Company montrent leur enthousiasme envers leur voyage dans le but de collecter les richesses de la terre nouvelle. Les protagonistes principaux sont Pocahontas et John Smith. La première est la fille du chef de la tribu Powhatan. Elle aime se promener librement partout dans les forêts. Quand le bateau de John Smith et ses compagnons accoste la terre de son peuple, elle s'intéresse à les découvrir. Le capitaine John Smith fait la connaissance de Pocahontas. Les deux tombent (Traduction des chansons dans le film...) Dr. Samah Abdou Nasr. 


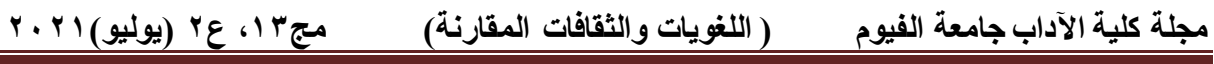

amoureux mais seront affrontés par les différences de cultures opposant leurs deux peuples.

Chaque groupe a ses propres convictions et ses motivations pour jouir de son existence sur terre. D'une part, la tribu des Powhatan admire la nature et respecte ses lois, ce qui se répète dans la chanson qui les présente. La nature leur ordonne un mode de vie auquel tout le monde se soumet avec plaisir.

"C'est la voix de la raison / Qui rythme chaque saison / En raisonnant dans nos âmes / Au son calme du tam-tam »

D'autre part, les colons britanniques n'ont qu'une seule obsession la chasse à l'or. Leur raisonnement est matériel.

"Sur les plages de Virginie / Les galets sont diamants / Le sable d'or est infini / Les rivières sont d'argent / Une petite pépite pour ma mie / Une petite pépite pour moi / Et toutes les autres seront pour toi / Virginia Compagnie. "Chanson : Au son calme du tam tam (Pocahontas, 1995)

Au début du film, les Amérindiens apparaissent en chantant en chœur une chanson du patrimoine pour célébrer la moisson. Puis c'est l'arrivée du chef de la tribu et des jeunes qui reviennent victorieux d'une mission pour défendre leur village des ennemis. C'est un rythme cadencé pour indiquer les rituels d'une vie calme et sereine, celle d'un peuple qui cultive et jouit de la nature. Le sacré est désigné par great spirit, traduit en français par Grand esprit de l'univers, Maître de l'ordre et du temps. Mais en arabe

(Traduction des chansons dans le film...) Dr. Samah Abdou Nasr. 
c'est Dieu.

\begin{tabular}{|c|c|c|}
\hline Version originale & Version française & Version arabe \\
\hline $\begin{array}{l}\text { O great spirit hear our } \\
\text { song } \\
\text { Help us keep the ancient } \\
\text { ways Keep the sacred fire } \\
\text { strong } \\
\text { Walk in balance all our } \\
\text { days }\end{array}$ & $\begin{array}{l}\text { Grand esprit de l'univers } \\
\text { Maître de l'ordre et du } \\
\text { temps } \\
\text { Garde le feu millénaire } \\
\text { Dans le coeur de tes } \\
\text { enfants }\end{array}$ & 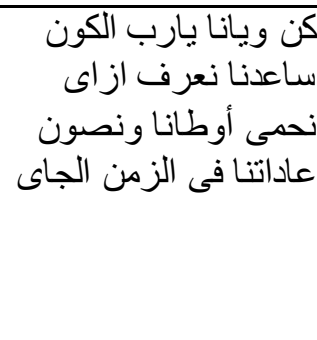 \\
\hline
\end{tabular}

Chanson : Au son calme du tam tam (Pocahontas, 1995)

Dans le doublage arabe, il n'est pas question de feu sacré car le feu est la marque de l'enfer ou du malheur dans la culture arabe. L'adaptateur choisit de le remplacer par une prière demandant l'aide, de Dieu afin de préserver et de défendre leur patrie et conserver leurs coutumes dans l'avenir. La protection de la terre natale ou de la patrie est clairement déclarée en plus de la préservation des coutumes sont les deux principes qui motivent le groupe des Amérindiens.

En français, l'accent est mis sur la protection des membres de la tribu en tant qu'enfants et la traduction est fidèle à l'anglais car le mot Grand esprit est acceptable mais il y a une phrase qui a été insérée pour suivre la musicalité des rimes Maître de l'ordre et $d u$ temps; ce qui est une réponse à la contrainte phonétique (garder les rimes temps / enfants) et en même temps un rappel du pouvoir de garder leurs rituels pour rendre le sens de «Help us

(Traduction des chansons dans le film...) Dr. Samah Abdou Nasr. 


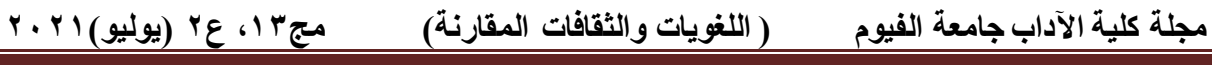

keep the ancient ways ».

En ce qui concerne les référents culturels, la chanson doublée maintient le refrain original prononcé en langue indienne des autochtones. Leurs habitudes, leurs mouvements, leurs maisons, leurs vêtements et leur rythme de vie est décrit par l'image et les détails dans la chanson. "Seasons go and Seasons come, Steady as the beating drum».

\subsection{La rencontre des deux protagonistes principaux}

Les différences de culture sont abordées comme raison de conflit selon la vision ethnocentrique. La remise en jeu de la définition du mot sauvage dans les paroles de Pocahontas. Les valeurs humaines sont évoquées selon son point de vue. Dans la traduction arabe, le mot sauvage a été traduit de deux façons différentes selon la situation de communication. L'adjectif متوحشة est l'équivalent du sens suivant : qui est le contraire de civilisé pour qualifier Pocahontas tel que la voit John Smith, mais c'est elle qui doit à son tour lui expliquer le vrai sens de la vie dans la chanson intitulée «L'air du vent». La version anglaise originale présente l'opposition des deux interlocuteurs sous forme de dialogue sans lui donner la chance de répondre. L'adaptation française oppose les pronoms $j e$ et tu pour enfin l'inviter à jouir de la vie avec l'emploi du nous. «Courrons dans les forêts d'or et de lumière. Partageons-nous les fruits mûrs de la vie ». Face à

(Traduction des chansons dans le film...) Dr. Samah Abdou Nasr. 
une description magique de la découverte de la forêt, l'image colorée correspondante est formée du mouvement de la caméra qui accompagne les deux protagonistes pleins de vie et d'enthousiasme pour suivre le vent et le peindre en mille couleurs.

C'est Pocahontas vs John Smith ou la sauvage vs le conquérant dit civilisé. Elle est motivée par son amour de son milieu naturel, elle parle selon ses émotions qui la guident tout au long de sa vie. Son raisonnement est présenté à travers sa visualisation d'elle-même, de son entourage et des êtres qui sont naturellement intégrés dans son quotidien. En français, elle utilise la métaphore "tes yeux sont remplis de nuages » pour critiquer sa prise de position, alors qu'en arabe la réponse à cet adjectif sauvage est une attaque sous forme de mots explicites dont la traduction serait : «Tu me prends pour ignorante sauvage ; ta race est supérieure ; dis donc conard, pourquoi t'es si arrogant? Dismoi que sais-tu dans ce monde ? Que sais-tu ?»

\begin{tabular}{|c|c|c|}
\hline Chanson originale & Version française & Version arabe \\
\hline $\begin{array}{l}\text { You think I'm an ignorant } \\
\text { savage } \\
\text { And you've been so many } \\
\text { places } \\
\text { I guess it must be so } \\
\text { But still I cannot see } \\
\text { If the savage one is me } \\
\text { How can there be so } \\
\text { much that you don't know } \\
\text { You don't know }\end{array}$ & $\begin{array}{l}\text { Pour toi je suis l'ignorante } \\
\text { sauvage } \\
\text { Tu me parles de ma } \\
\text { différence } \\
\text { Je crois sans malveillance } \\
\text { Mais si dans ton langage } \\
\text { Tu emploies le mot sauvage } \\
\text { C'est que tes yeux sont } \\
\text { remplis de nuages } \\
\text { De nuages }\end{array}$ & 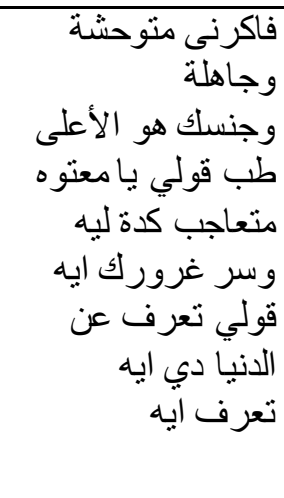 \\
\hline
\end{tabular}

(Traduction des chansons dans le film...) Dr. Samah Abdou Nasr. 
Chanson : L'Air du vent (Pocahontas, 1995)

Le rapport avec l'autre est l'un des points que Pocahontas soulève dans cette chanson: la restriction dans la version française convient à indiquer le caractère ethnocentrique de John Smith et de ses compagnons; or il devrait changer d'avis et se questionner dans la version française. L'interrogation en tant que structure ne figurait pas dans l'original anglais. En arabe, c'est plus catégorique en utilisant l'impératif dont la traduction est la suivante : essaye de marcher seul dans cette vie, beaucoup de gens t'apprendront!

\begin{tabular}{|c|c|c|}
\hline Chanson originale & Version française & Version arabe \\
\hline $\begin{array}{l}\text { You think the only people } \\
\text { who are people } \\
\text { Are the people who look } \\
\text { and think like you } \\
\text { But if you walk the } \\
\text { footsteps of a stranger } \\
\text { You'll learn things you } \\
\text { never knew you never } \\
\text { knew }\end{array}$ & $\begin{array}{l}\text { Pour toi l'étranger ne } \\
\text { porte le nom d'homme } \\
\text { Que s'il te ressemble et } \\
\text { pense à ta façon } \\
\text { Mais en marchant dans } \\
\text { ses pas tu te questionnes } \\
\text { Es-tu sûr au fond de toi } \\
\text { d'avoir raison? }\end{array}$ & 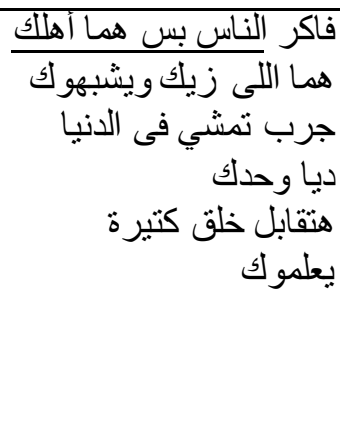 \\
\hline
\end{tabular}

Chanson : L'Air du vent (Pocahontas, 1995)

Dans la chanson des Sauvages, l'autre est désigné par des termes qui affichent l'animosité et le conflit humain. Les connotations péjoratives de certains mots reflètent les sentiments de sous-estime et la haine des deux parties, ce que nous cherchons à observer de près.

(Traduction des chansons dans le film...) Dr. Samah Abdou Nasr. 


\subsection{Le conflit}

John Smith est enlevé par les hommes de la tribu de Pocahontas. C'est l'heure du déclenchement des hostilités ou l'annonce de la guerre. Ratcliffe, le commandant du groupe de colons incite ses marins armés à sauver Smith alors que l'autre groupe des amérindiens se préparent à le tuer et à affronter ces conquérants étrangers. Nommer la différence de race et s'attaquer verbalement à l'autre qui est qualifié de sauvage. Cette dénomination répétée des deux parties indique le refus de la diversité culturelle. En effet, la définition encyclopédique du mot «sauvage (l'adjectif latin silvester désigne tout ce qui est « de la forêt ») rejette dans une catégorie de l'infrahumain des individus et des sociétés auxquelles on attribue un genre de vie qui les rapproche plus de la vie animale que de la culture humaine. » Le terme sauvage est le même en anglais et en français mais en arabe il est traduit par les animaux féroces (الوحوش). Selon 1'Encyclopedia Universalis en ligne, le refus de la diversité des cultures se manifeste de deux façons: tendance à refuser l'humanité de l'autre et essayer de l'assimiler ou le façonner à sa manière. Entre oralité et vulgarité, la violence verbale augmente avec le rythme de la chanson et remonte jusqu'à l'explosion (voir annexe); les exemples suivants illustrent les expressions que les deux groupes utilisent pour nommer l'adversaire. Les mots de la version originale réfèrent à une discrimination des deux côtés, ce qui sera

(Traduction des chansons dans le film...) Dr. Samah Abdou Nasr. 
transmis dans les traductions. Premièrement, la couleur de la peau est évoquée, puis la religion et enfin le manque d'éthique et de scrupule. La version anglaise originale, les Amérindiens sont définis en tant que «filthy little heathens; their whole disgusting race is like a curse ». En français, ils sont des païens, des bêtes, des bons à rien, et de la vermine. Ces appellatifs dépréciatifs justifient leur extermination par les Blancs qui dans la version arabe, ont utilisé les mots suivants pour désigner leurs adversaires jugés comme sauvages:

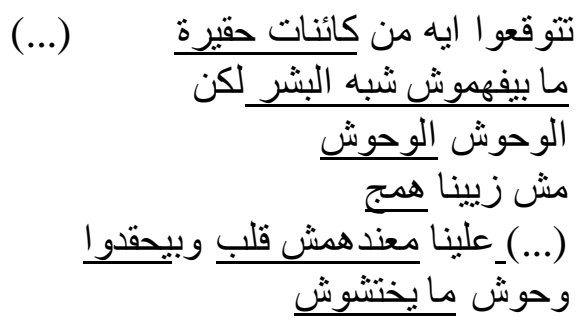

Traduction de la chanson : Savages (Pocahontas, 1995)

A l'opposé, les Indiens disent que ces hommes blancs sont des démons (qui) sèment la mort sans remords. Les sonorités sont spécifiques à la version française. Les termes péjoratifs en arabe égyptien sont multiples et marquent le non-respect mutuel. Ce sont des mots vulgaires qui riment avec les syllabes homéotéleutes) et qui veulent dire salaud et salopard ( زبالة - حثالة). Le capitaine Ratcliffe donne ses ordres de tuer les «barbares » comme si c'était la justice : Allez les gars, tuez les barbares, c'est la justice! C'est légal de les battre!

(Traduction des chansons dans le film...) Dr. Samah Abdou Nasr. 


$$
\text { 》 اتجمعو ا يا رجالة / قتل الهمج عدالة/ حلال فيهم الضرب 《 }
$$

Du point de vue des Amérindiens, le Blanc représenté par les conquérants est violemment critiqué et dévalorisé : le groupe tripartite sans pitié, sans scrupule, sans honnêteté qualifie les ennemis, ce qui s'est traduit en arabe égyptien par l'expression suivante «رحة ضمير ذمة معندوش》. Les arguments avancés sont typiquement révélateurs de la culture égyptienne y compris les appellations et les preuves : les colons sont désignés comme étant des êtres maléfiques en guerre contre le bien, vindicatifs dangereux, fils de Satan, indignes de confiance. Le mal et la méchanceté sont qualifiés en rapport avec le poison en arabe égyptien; le paroxysme est atteint à travers la métonymie mettant en cause cette ressemblance aux descendants de Satan. L'évocation de pot-de -vin indique l'image négative des humains qui sont vraiment malhonnêtes dans leur course à l'argent.

$$
\text { برشى ضد الخير / سماوي خطير / أبناء الثشطان جنس مالوش أمان }
$$

La musicalité impose l'emploi des mots soulignés pour respecter le choix des rimes. Le même principe préside au choix de « raison/démon; encore/or » dans la version française.

Nous avions raison,

L'homme blanc est un démon.

Le seul dieu qu'il adore encore, C'est l'or!

Chanson : Des sauvages (Pocahontas, 1995)

(Traduction des chansons dans le film...) Dr. Samah Abdou Nasr. 
De plus, l'absence de religion est déplorée étant donné que l'homme blanc est sans cour et sans foi « معندش ايمان ومايعرفش ». De nouveau la couleur de la peau est évoquée pour caractériser la cruauté malgré l'apparence de la couleur blanche représenté par le lait dans la culture arabe :

$$
\text { دهوش لون اللبنوتحته غدر الزمن لإذا فهما إذن وحوش }
$$

L'emploi des deux connecteurs de conséquence c'est pourquoi (لذإ) et donc (إذن) dans la phrase suivante ne fait que confirmer la méchanceté et le mal incarné par ces étrangers. L'anglais utilise deux mots pale face et milky et a recours à l'interrogation pour se moquer de leurs caractéristiques humaines.

Beneath that milky hide

There's emptiness inside

I wonder if they even bleed

Chanson : Savages (Pocahontas, 1995)

Or, la version française l'expression race de vipères désigne les Amérindiens qui aux yeux des Britanniques doivent être tués car ils ne leur ressemblent pas ! Vu qu'ils n'ont pas la même couleur de peau, le capitaine Ratcliffe annonce: "Je les extermine »!

Puisqu'ils ne sont pas blancs, Ils sontforcément méchants

En français, l'allitération du son 's' dans les mots «seul, c'est, dessous, sa, lys, vice » rappelle le son du serpent et en

(Traduction des chansons dans le film...) Dr. Samah Abdou Nasr. 


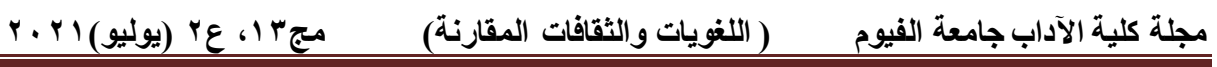

même temps la méchanceté et le danger que représentent les colons pour les autochtones.

Dessous sa peau de lys, ses vices se glissent.

Ils sèment la mort sans remords!

Le refrain qui est répété des deux groupes est l'appel à la guerre contre ces sauvages. D'où l'expression «Now we sound the drums of war! » Battons les tambours de la guerre!

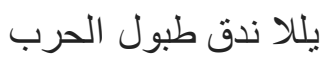

Les Amériendiens terminent leurs paroles par une prière afin de vaincre l'ennemi: l'appel à la confrontation s'unit à la confiance en Dieu.

$$
\text { يلا ندق طبول الحرب / بارك لنا يارب / بلا ندق طبول الحرب }
$$

Face à cette guerre, Pocahontas court et fait de son mieux pour l'arrêter et elle arrive au bon moment pour dire non à sa tribu et essayer de sauver John Smith.

\section{Procédés d'adaptation}

\subsection{La domestication}

Dans la traduction audiovisuelle, l'adaptation englobe la possibilité de changer des «anthroponymes ou de toponymes réputés méconnus du spectateur-type.» Brisset (2017 : 38). Examinons la traduction vers l'arabe, la démarche suivie, dès le titre, consiste à adapter le sous-titre qui définit la héroïne ; une

(Traduction des chansons dans le film...) Dr. Samah Abdou Nasr. 
légende indienne devient princesse de la forêt. Les noms des éléments naturels dans la nature dans la vie des Améridiens tels que grinning Bob Cat, Blue Corn moon, the heron sont rendus par d'autres mots comme montre le tableau suivant, alors qu'il se sert de l'étoffement en gardant le nom du Sycamore qui est spécifique des fôrets et qui apparaît clairement dans la scène ( l'arbre du sycamore شجر السيكامور ).

\begin{tabular}{|c|c|c|}
\hline Version anglaise & Version française & Version arabe \\
\hline $\begin{array}{l}\text { - The rainstorm and } \\
\text { the river are my } \\
\text { brothers } \\
\text { The heron and the } \\
\text { otter are my friends }\end{array}$ & $\begin{array}{l}\text { Je suis fille des torrents, } \\
\text { sœur des rivières } \\
\text { La loutre et le héron } \\
\text { sont mes amis }\end{array}$ & الثير أخت النهر و والثجر دول \\
\hline $\begin{array}{l}\text { - Have you ever } \\
\text { heard the woolf } \\
\text { crying to the blue } \\
\text { corn moon } \\
\text { Or asked the } \\
\text { grinning bobcat why } \\
\text { he grinned }\end{array}$ & $\begin{array}{l}\text { Comprends-tu le chant } \\
\text { d'espoir du loup qui } \\
\text { meurt d'amour } \\
\text { Les pleurs du chat } \\
\text { sauvage au petit jour? }\end{array}$ & 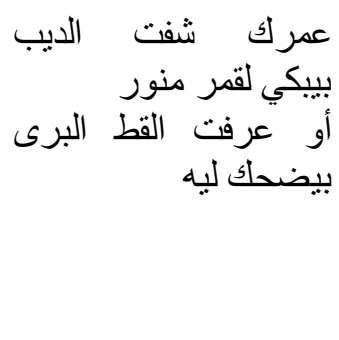 \\
\hline
\end{tabular}

Chanson : L'Air du vent (Pocahontas, 1995)

Le loutre et le héron deux animaux qui ne se trouvent pas dans la culture du public égyptien et arabophone, alors pourquoi ne pas les remplacer par le soleil et la lune, deux éléments appréciés dans l'imaginaire du monde arabe et faisant l'objet d'une multitude de chansons ? Sur le plan syntaxique, les

(Traduction des chansons dans le film...) Dr. Samah Abdou Nasr. 
traductions française et arabe suivent la forme d'un prédicat en changeant l'ordre de l'original. La description fille et sœur est rendue, selon le procédé d'omission, par sœur en arabe. Selon ce même procédé d'omission, la différence de couleur de peau n'a pas été évoquée dans cette chanson; la phrase For whether we are white or copper-skinned est complètement absente de la version arabe. Elle est substituée par une phrase causale qui complète le sens de peindre en mille couleurs l'air du vent (en arabe c'est plus و عشان ما يقى كل شئ (pour que tout soit beau) et plus direct : pour جميل

Par contre, le procédé d'ajout est utilisé en français : le loup pleure dans la chanson en anglais mais se transforme en chant d'espoir. En plus, l'amour est ajouté pour la mesure des vers de la chanson, ce mot rime avec jour. La lune devient éclairée en arabe pour que l'adjectif منور rime avec le verbe (peux-tu?)

Friends en anglais devient amis en français, mais des voisins en arabe. Il existe une chanson libanaise très connue de Fayrouz intitulée la lune et moi, nous sommes voisins, il se peut que le traducteur soit influencé par cette idée. Souvent on a recours à des éléments du patrimoine culturel qui nous imprègnent inconsciemment.

(Traduction des chansons dans le film...) Dr. Samah Abdou Nasr. 
Par ailleurs, le traducteur garde les phrases énoncées en amérindien où l'oralité est à l'honneur. Par exemple la phrase qui signifie au son calme des tam-tam. "Hega hega yam pi ye he hega». En arabe, le son du tambour est rendu par une onomatopée adéquate à l'arabe صوت الطبل بيقول دوم. Chanson : Au son calme du tam tam (Pocahontas, 1995)

Dans une autre chanson «Ecoute ton cœur », la phrase que que natura est maintenue car c'est la mère feuillage, l'arbre qui incarne l'âme de la grand-mère de Pocahontas, qui prononce ces mots. Le message met en relief la nécessité de se laisser guider par ses sentiments et les éléments de la nature surtout le vent.

\subsection{Evocation du rapport à la terre d'une culture à l'autre}

La terre est un facteur essentiel dans notre rapport à la vie et à Dieu; l'Homme doit laisser une trace sur terre par ses actes, alors quelle est la relation de chaque peuple envers la terre ? Les Amérindiens la protègent et la respectent pendant que les colons veulent la conquérir. Cette idée de posséder la terre est le rêve de Jon Smith et de son groupe. Mais Pocahontas lui explique que cette propriété ne doit pas être son seul souci, au contraire il devrait partir à la découverte de la beauté de la terre.

(Traduction des chansons dans le film...) Dr. Samah Abdou Nasr. 


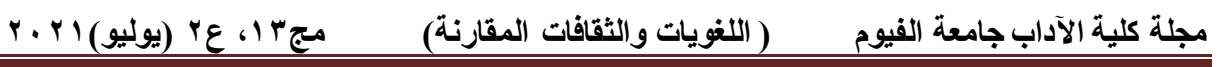

"Mais la terre n'est que poussière / Tant que l'homme ignore comment / Il peut peindre aux mille couleurs l'air du vent». chanson : L'air du vent (Pocahontas, 1995)

Dans la version arabe, Dieu est omniprésent dans les paroles des Amérindiens. La terre est la propriété de Dieu qui l'a remplie de richesses de toute sorte. "دى ما يملكها (اوحده غير الله

A la fin de cette évocation de la terre c'est une invitation qui fait appel à un dicton arabe connu املك من الدنيا ما شئت ،لكنك ستخرج منها كما جئت (tu peux posséder autant de terre mais tu vas quitter tout à la fin. En effet la traduction fait écho à cette phrase pour mettre en valeur la métaphore peindre le vent.

$$
\text { . املك من الأرض وكتر هتلاقى تراب مش أكتر لحدماترسم بجميع ألوان الرياح. }
$$

Dans sa démarche, «le tradapteur fonctionne par associations d'idées et s'appuie sur les clichés, les idiomes, les champs lexicaux et les collocations de sa langue. » Guillemain (2019).

Dans les deux versions française et arabe, l'utilisation des tournures stylistiques sous forme d'idiomes est récurrente. Mais quel effet ont ces idiomes sur le spectateur? C'est une sorte de réécriture dans le cadre de l'effet voulu par l'original. La création consiste à gérer les équivalences en communicant le message et

(Traduction des chansons dans le film...) Dr. Samah Abdou Nasr. 


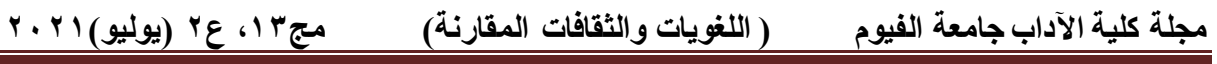

laissant une empreinte sonore dans la mémoire du public.

\subsection{Emploi de termes et tournures en adéquation avec le registre familier}

La création dans l'adaptation à la culture égyptienne se révèle au niveau lexical dans l'emploi de termes du registre familier qui conviennent à la musique en rapport avec la situation.

« For glory, God and gold and the Virginia Company»

$$
\text { 《عيشة هنية مع حرية مع شركة فرجينيا ( ع) }
$$

Chanson : Virginia Company (Pocahontas, 1995)

Notons l'ajout de quelques phrases, juste pour donner des équivalents qui riment avec les syllabes précédentes sous forme de dictons ; par exemple cette phrase (مباح لو تطلب تتول) est forgée sur un autre proverbe connu en arabe dialectal puisqu' on dit celui qui demande une chose peut l'avoir اللي يطلب ينول . Les chansons sont pleines de sentences telles que le bonheur ici-bas n'a pas de prix; nous tournons tous ensemble au fil des jours dans un cercle, une ronde à l'infini.

En effet, les paroles de Pocahontas témoignent d'une vision profonde, sentimentale et pleine de valeurs humaines émanant de sa bonté, son innocence et sa simplicité. Elle fascine John par sa vivacité et son enthousiasme. Sa vitesse de parole parfois traduit son déplacement libre et énergétique au milieu de

(Traduction des chansons dans le film...) Dr. Samah Abdou Nasr. 
la nature.

Elle connaît parfaitement son milieu préféré et cette connaissance est merveilleusement traduite par une expression familière ( dont la traduction littérale est la suivante : je connais ma terre pierre par pierre عار فاها طوبة طوبة ).

Nous pouvons conclure que l'adaptation se fait selon des contraintes du rythme musical des chansons. Ce qui oblige parfois à changer l'ordre des mots ou opter pour une autre structure stylistique. Les rimes priment concernant le choix des mots à la fin des vers. En fait, l'emploi de certaines tournures ou l'inversion de l'ordre des phrases selon les contraintes syntaxiques de la langue cible offrent la possibilité de restituer les lyrics des chansons en adéquation avec la musique. Le choix des mots dépend des syllabes finales recherchées, ce qui permet de garder parfois le rythme en ajoutant une tout autre phrase absente de l'original pour retrouver la même tonalité.

La désignation d'altérité et d'ethnocentrisme à travers le mot sauvage a permis de déceler des valeurs qui font la grandeur de l'Homme en réitérant l'idée de respecter les autres habitants de cette terre en plus des animaux et des plantes, implicitement cela est l'essence réelle de l'existence. L'exploration de la nouvelle terre devrait, au lieu de rechercher l'or, voir de près les particularismes culturels de chaque région autour de la planète. La

(Traduction des chansons dans le film...) Dr. Samah Abdou Nasr. 
répétition de certaines parties de phrases est typique dans les chansons pour créer l'effet émotionnel à travers ces anaphores, ces dernières restent dans l'esprit de l'audience et surtout lorsqu'il s'agit d'une interrogation qui incite à agir. En ce qui concerne la langue familière en arabe égyptien, l'analyse a repéré plusieurs tournures stylistiques typiques à la culture cible ce qui est motivé par la garantie d'une bonne réception du film. Les aspects phraséologiques de certains idiomes ne font qu'augmenter cette réception basée sur une familiarité du lexique et des effets qu'elle crée chez le public.

Au plan culturel, la critique de la vision de John Smith et de ses copains ont remis en cause la matérialité de cette perception du monde et le rejet de tout ce qui est différent tout en mettant en lumière le besoin d'éveil des sens ainsi que le retour à la vie naturelle. Il reste à mesurer à quel point cette peinture de personnages exotiques correspond aux attentes des spectateurs. La diffusion récente des vidéos de ces chansons dans les réseaux sociaux fait revivre le film et il serait intéressant de voir les commentaires vis-à-vis des choix des adaptateurs.

(Traduction des chansons dans le film...) Dr. Samah Abdou Nasr. 


\section{Bibliographie}

Michel Ballard, M. (2006). « À propos des procédés de traduction », in Palimpsestes, Hors série, mis en ligne le 01 septembre 2008, DOI : https://doi.org/10.4000/palimpsestes.386

Ballard, M. (2004). Le commentaire de traduction anglaise, Paris, Nathan, coll. «128 », première édition 1992.

Ballard, M. (1997). «Créativité et traduction», in Target 9:1 (1997), 85-110.

https://doi.org/10.1075/target.9.1.06bal

Bastin, G. (1993). « La notion d'adaptation en traduction. », in Meta, vol. 38, no. 3, 473-478.

Bell, A. (1984). «Language style as audience design », in Language in Society, 13(2), 145-204.

doi:10.1017/S004740450001037X

Brisset, F. (2017). « Le doublage, à la frontière entre traduction et adaptation?», in TranscUlturAl, vol. 9.2 (2017), 32-46. http://ejournals.library.ualberta.ca/index.php/TC

Dąbek, K. (2018). «Exigences formelles et transition sémantique dans la traduction des chansons filmiques », in ROCZNIKI HUMANISTYCZNE, Tome LXVI, zeszyt 5 - 2018. http://dx.doi.org/10.18290/rh.2018.66.5-12

(Traduction des chansons dans le film...) Dr. Samah Abdou Nasr. 
Dastugue, G. (2018). «La chanson chez Disney une stratégie en chanté », in Inter-Lignes, (21), 111-124.

Defacq, A. (2011). Les parasites de traduction : entre adaptation et fidélité. L'exemple des comédies musicales américaines, Thèse de doctorat, Université d'Angers. https://tel.archivesouvertes.fr/tel-00972046

Franzon, J. (2008). «Choices in Song Translation», in The Translator, 14, 373-399.

DOI : 10.1080/13556509.2008.10799263

Encyclopedia Universalis en ligne

https://www.universalis.fr/

Guillemain, A. (2019). «Chansons pop: tradapter pour que résonne le sens », in Traduire, $240 \mid 2019$.

http://journals.openedition.org/traduire/1738

Lemieux, R. (2010). Éthique et esthétique de l'Autre en traduction: une réflexion à partir de récentes critiques contre la traductologie d'Antoine Berman, in Translationes 2 (2010), 39 52

https://doi.org/10.2478/tran-2014-0024

Moriceau, J. (2012). « La translation. Aspects de la traduction de films, sous-titres et doublage, conditions et conditionnement. », in

(Traduction des chansons dans le film...) Dr. Samah Abdou Nasr. 
Le cinéma parle! Études sur le verbe et la voix dans le cinéma anglophone, CICLAHO, vol. 6, 2012, 193-206

Paillard, M. (2000). Lexicologie contrastive, anglais-français, Paris, Ophrys.

Paquin, R. (1998). « Translator, Adapter, Screenwriter Translating for the Audiovisual. », in Translation Journal, vol. 2, no. 3, 1998. https://translationjournal.net/journal/05dubb.htm

Plourde, E. (2002). «The Dubbing of the Simpsons. Cultural Appropriation, Discursive Manipulation and Divergences. ", in Texas Linguistic Forum, vol. 44, no. 1, 2002, 114-131.

Richet, B. (2004). « On connaît la chanson »: Aspects de la traduction des chansons dans la bande dessinée Astérix, in Les Cahiers de l'ILCEA, ELLUG, 2004, 151-179 https://halshs.archives-ouvertes.fr/halshs-00661980

Yvane, J. (1996). «Le doublage filmique : fondements et effets », in Yves Gambier (éditeur), Les transferts linguistiques dans les media audiovisuels, Villeneuve-d'Ascq, Presses Universitaires du Septentrion, 133-143.

\section{Corpus des chansons :}

Transcription des chansons françaises du corpus https://paroles2chansons.lemonde.fr/paroles-pocahontas consulté le 11/07/2020

(Traduction des chansons dans le film...) Dr. Samah Abdou Nasr. 
Transcription des chansons anglaises du corpus

https://www.disneyclips.com/lyrics/pocahontas.html

consulté le 10/07/2020

Transcription des chansons arabes du corpus

Faite par nous-même.

\section{Titre des chansons}

Virginie compagnie ou La Compagnie de la Virginie (The Virginia Company) - Chœurs (chanson française), consulté le $06 / 06 / 2020$

https://www.youtube.com/watch?v=62KMuYhYCP4

Virginia compagny (chanson anglaise) - Smith \& Chœurs, consulté le 06/06/2020

https://www.youtube.com/watch?v=Ne2tzfxQ6T4

Virginia compagny (chanson arabe) Smith \& Chøurs شركة فرجينيا , consulté le 06/06/2020

https://www.youtube.com/watch?v=ZDN2m-372i0

Steady As The Beating Drum - Pocahontas (chanson anglaise), consulté le 08/06/2020

https://www.youtube.com/watch?v=Y5 YHzR57u4 A

Steady As The Beating Drum - Pocahontas (chanson arabe),

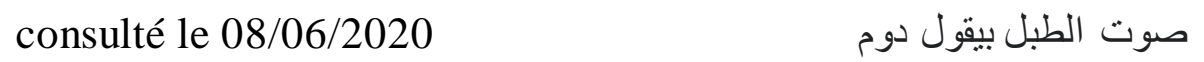
https://www.youtube.com/watch?v=h579pLXc4RY Au son calme des tam-tams (Steady As The Beating Drum) Chœurs, consulté le 08/06/2020

(Traduction des chansons dans le film...) Dr. Samah Abdou Nasr. 
https://www.youtube.com/watch?v=30QVUgPY73M

Colors Of The Wind (chanson anglaise) Pocahontas, consulté le $15 / 06 / 2020$

https://www.youtube.com/watch?v=O9MvdMqKvpU

Colors Of The Wind (chanson arabe) Pocahontas, consulté ألوان الرياح 15/06/2020

https://www.youtube.com/watch? $v=f \_5 v q T b O D C 8$

L'Air du vent (Colors Of The Wind) - Pocahontas (chanson française), consulté le 15/06/2020

https://www.youtube.com/watch?v=OAhCfI5hLjU

Savages Pocahontas, Powathan, Ratcliff \& Chœurs (chanson anglaise), consulté le 19/06/2020

https://www.youtube.com/watch?v=2av9SQs MIi8

Savages (chanson arabe) Pocahontas, Powathan, Ratcliff \& Chœurs consulté le 19/06/2020

https://www.youtube.com/watch?v=HZlAU7JJEsI

Des sauvages (chanson française) - Pocahontas, Powathan, Ratcliff \& Chœurs, consulté le 19/06/2020

https://www.youtube.com/watch?v=COv-1 mpFVeU

Ecoute ton Cour - Pocahontas (chanson française), consulté le $21 / 06 / 2020$

https://www.youtube.com/watch?v=YkzMDobun-I

Listen With Your Heart - Pocahontas (chanson arabe) اسمعيه بقلبك

(Traduction des chansons dans le film...) Dr. Samah Abdou Nasr. 


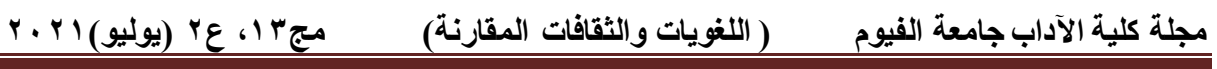

consulté le 21/06/2020

https://www.youtube.com/watch?v=4sI--X91Bi0

Listen With Your Heart Pocahontas, consulté le 21/06/2020

https://www.youtube.com/watch?v=sm0uR81AZhA

(Traduction des chansons dans le film...) Dr. Samah Abdou Nasr. 


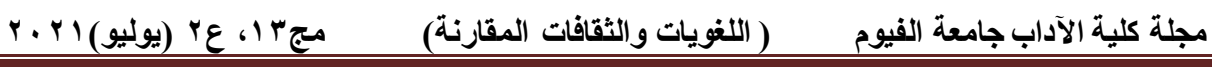

\section{Translating the songs of Disney's animated film \\ Pocahontas between adaptation and music}

\section{Abstract}

This article aims to discover the translation of the songs of the Disney cartoon film Pocahontas into French and Egyptian Arabic. Why choose the Egyptian dialect for Arabic translation? Between dialect and classical Arabic, what are the choices to translating for audience in the Middle East? We aim to discover the processes of transfer of cultural references: connotations (name the difference of races for example), the moral value of some specific cultural phenomena (cultural heritage), the sacred, etc.

Dubbing the film's songs in Egyptian colloquial Arabic dialect is examined in comparison to original songs and French version. Cultural differences are represented in this film by two opposed groups: indigenous people of America and British invaders who try to gain fortune. Differences in cultures appear in various ways through the group's relationship to the external environment, to other people and to the land. Beliefs, values and practices are compared from the beginning till the end.

(Traduction des chansons dans le film...) Dr. Samah Abdou Nasr. 